\title{
Argumentación en la matemática escolar infantil: Análisis de una actividad STEM usando la Situación Argumentativa en Conexión Interdisciplinar
}

\author{
Ángel Alsina, Universidad de Girona (España) \\ Claudia Cornejo-Morales, Pontificia Universidad Católica de Valparaíso (Chile) \\ María Salgado, Universidad de Santiago de Compostela (España)
}

\begin{abstract}
Argumentación en la matemática escolar infantil: Análisis de una actividad STEM usando la Situación Argumentativa en Conexión Interdisciplinar
\end{abstract}

\section{Resumen}

Se analiza la presencia de la argumentación en una actividad STEM implementada con 20 alumnos de 3. de Educación Infantil (5-6 años). Dado el carácter interdisciplinar de la actividad, en la que se integran conocimientos de matemáticas y ciencias, para realizar el análisis se usa una variante de la Situación Argumentativa (SA), que considera aspectos contextuales y funcionales de la argumentación en la matemática escolar de las primeras edades: la Situación Argumentativa en Conexión interdisciplinar (SAC). El análisis de los datos muestra que, en la secuencia de tareas, la principal función de la argumentación es explicar y el carácter es narrativo: los argumentos son breves y concisos, responden a una pregunta específica y usan expresiones clave para mostrar la relación entre sus elementos constitutivos. Se concluye que es necesario diseñar nuevos estudios con este modelo de análisis para consolidar estos resultados iniciales.

Palabras clave. Argumentación en matemáticas; Situación Argumentativa; interdisciplinariedad; STEM; Educación Infantil.

Argumentation in early school mathematics: Analysis of a STEM activity using the Argumentative Situation in Interdisciplinary Connection

\section{Abstract}

The presence of argumentation in a STEM activity implemented with 20 students of Early Childhood Education Grade 3 (5-6 years) is analysed. According to the interdisciplinary nature of the activity, where knowledge of mathematics and science is integrated, a variant of the Argumentative Situation, which considers both functional and contextual aspects of argumentation in early school mathematics, is used: the Argumentative Situation in Interdisciplinary Connection. The analysis of data shows that in the sequence of tasks, the main function of the argumentation is to explain and the character is narrative: the arguments are short and concise, they answer a specific question and use key expressions to show the relationship between its constituent elements. It is concluded the necessity of designing new studies with this analytical framework to consolidate these initial results.

Keywords. Argumentation in mathematics; Argumentative Situation; interdisciplinarity; STEM; Early Childhood Education.

\section{Introducción}

La visión de la enseñanza de las matemáticas que predomina en las orientaciones curriculares contemporáneas puede ubicarse dentro del grupo de teorías denominadas InquiryBased Education, sobre el aprendizaje por indagación de los alumnos con apoyo subsidiario 
del profesorado (Artigue y Blomhøj, 2013). Este planteamiento puede malinterpretarse al entender que, dado que los alumnos aprenden "con poca guía por parte del profesor" (Godino et al., 2015, p. 250), aprenden más o menos solos, sin tener en cuenta el papel del profesorado en la enseñanza. En este sentido, diversos autores y organismos se refieren a la gestión de la enseñanza de los contenidos a través de los procesos matemáticos de resolución de problemas, modelización, razonamiento, argumentación, comunicación, conexiones, representación, etc. (e. g. Alsina et al., 2021; NCTM, 2003; Niss, 2002). Aunque estos procesos, en su conjunto, son esenciales para promover el desarrollo de la indagación matemática en clase, este estudio se centra en la argumentación en clase de matemáticas de Educación Infantil, por su papel en el desarrollo del pensamiento matemático y en la formación de ciudadanos autónomos, críticos y reflexivos desde las primeras edades (NCTM, 2003).

Cornejo-Morales et al. (2021) señalan diversas perspectivas teóricas desde las que se han investigado y caracterizado los elementos de la argumentación (Boero, 2011; Toulmin, 1958) y de la argumentación en matemáticas. Estas perspectivas han considerado aspectos: a) estructurales (Inglis et al., 2007; Knipping, 2008; Solar y Deulofeu, 2016); b) lógico-semánticos (Balacheff, 1999; De Villiers, 1993; Duval, 1999); y c) sociales (Boero et al., 2010; Douek, 2007; Goizueta, 2019; Goizueta y Solar, 2019; Krummheuer, 1995). Esta diversidad de aproximaciones, aunque enriquecen el campo, abordan el análisis de la argumentación focalizándose en su estructura y en los tipos de argumento, lo que no responde a las características de la argumentación en el aula de Educación Infantil. Aquí, los alumnos suelen expresar sus ideas usando frases breves, poco elaboradas y de manera no estructurada; o bien mediante acciones no verbales, cambiando de foco de interés rápidamente, a medida que reciben nuevos estímulos.

Para contribuir al desarrollo de estudios que analicen de forma conjunta diversos aspectos de la argumentación en las clases de matemáticas de Educación Infantil, en mayor sintonía con las orientaciones curriculares de esta etapa (Cornejo-Morales y Alsina, 2020), este estudio se enlaza con un trabajo preliminar en el que se describe un modelo de análisis integrador denominado Situación Argumentativa (SA), que considera aspectos contextuales y funcionales de la argumentación en las primeras edades (Cornejo-Morales et al., 2021). La SA emerge como modelo de análisis específico para las primeras edades sin descartar posibles aplicaciones en otras etapas. Por un lado, los elementos que considera la SA permiten definir de forma objetiva la actividad matemática del aula de infantil, poniendo el foco en la argumentación. Por otro lado, el abordaje multifactorial proporciona una visión holística de la actividad matemática que se genera en el aula de infantil. El análisis de conjunto permite observar la actividad matemática infantil como un todo integrado y global que determina cómo se comportan las partes que interactúan (Cornejo-Morales et al., 2021).

Considerando que los alumnos de Educación Infantil aprenden holísticamente, principalmente a partir de las conexiones entre conocimientos de distinta naturaleza, en este artículo se analiza la argumentación en el marco de una actividad STEM implementada en una clase de Educación Infantil en la que se integran conocimientos de matemáticas y ciencias. Dadas las características de la actividad, la principal novedad de este nuevo estudio es que para describir las características de la argumentación se utiliza como modelo de análisis una variante de la SA: la Situación Argumentativa en Conexión interdisciplinar (SAC), que considera conexiones entre disciplinas. Además de presentar un modelo de análisis holístico, se pretende contribuir a que el profesorado de las primeras edades disponga de un instrumento 
que oriente acerca de los elementos a considerar para promover la argumentación en la matemática escolar infantil.

\section{La argumentación en matemáticas}

Considerando las aproximaciones de la argumentación en matemáticas mencionadas en la introducción, y a fin de responder a las necesidades y características de los primeros años, se asume que la argumentación es "una actividad comunicativa y situada, por medio de la cual los niños y niñas entregan razones (para otros o para sí mismos) para justificar y convencer (o convencerse) sobre cierta posición o cuestionarla reflexivamente" (Cornejo-Morales et al., 2021, p. 161). Concebir la argumentación como actividad implica entenderla como medio para tender puentes entre procesos interpersonales e intrapersonales (Schwarz et al., 2003), y no como aplicación de habilidades generales para construir y evaluar argumentos, dar razones, construir un contraargumento o responderlo (Kuhn, 1991).

Desde esta visión, el elemento central de la argumentación es el argumento, que en Cornejo-Morales et al. (2021) se concibe como producto de aspectos cognitivos, emocionales y/o de conductas imitativas, entre otros, que corresponden tanto a producciones orales y escritas de niños y niñas para justificar o cuestionar algo, como a reconstrucciones lingüísticas a posteriori mediante las que ellos -o un observador-expresan sintéticamente el aspecto argumentativo de las producciones.

La Situación Argumentativa (SA) permite describir y caracterizar la argumentación en clase de matemáticas de las primeras edades mediante cinco elementos estructurales (Figura 1): 1) Argumento (¿qué se argumenta y por qué?); 2) Interacción (¿quiénes argumentan?); 3) Función (¿para qué se argumenta?); 4) Carácter (¿cómo se argumenta?); y 5) Matemática (¿sobre qué se argumenta?) (Cornejo-Morales et al., 2021, p. 165).

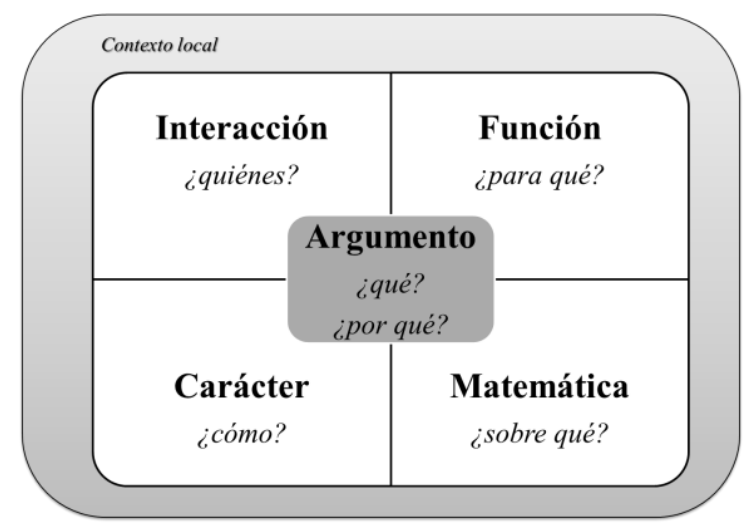

Figura 1. Representación de la Situación Argumentativa (SA)

Considerando la importancia de las conexiones en las aulas de los primeros años, se propone una variante de la SA. Esta variación responde a las características holísticas de la Educación Infantil e implica la ampliación de uno de los elementos estructurales de la SA, la Matemática, que se substituye por Conexiones interdisciplinares con el propósito de hacer hincapié en la integración de conocimientos de distintas disciplinas. La Figura 2 muestra una versión adaptada de la SA: la Situación Argumentativa en Conexión interdisciplinar (SAC). 


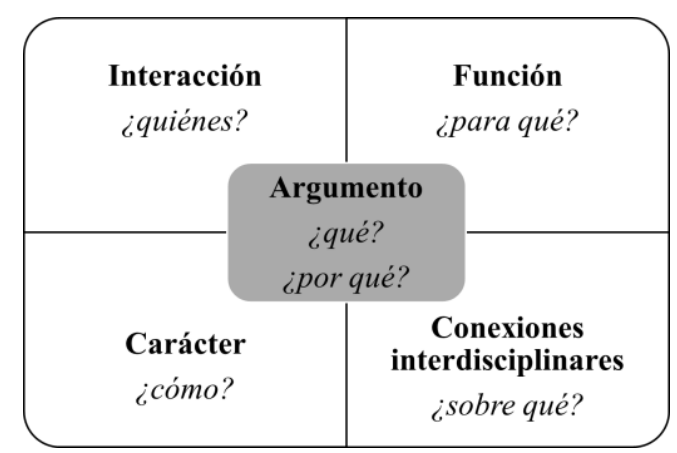

Figura 2. Representación de la Situación Argumentativa en Conexión interdisciplinar (SAC)

El Argumento responde a qué dicen y/o hacen los participantes de la interacción y por $q u e ́$ lo dicen y/o hacen. Tiene dos elementos clave: la posición adoptada respecto a una afirmación dada y las razones que dan cuenta de dicha posición.

La Interacción responde a quiénes intervienen en la argumentación. Puede ser de: 1) Trabajo en gran grupo, cuando el profesor interactúa con toda la clase; 2) Trabajo entre pares en pequeño grupo, cuando la interacción se entabla entre alumnos del grupo y sin intervención del profesor; 3) Trabajo en pequeño grupo con el profesor, cuando el profesor interactúa de manera privada con un grupo; y 4) Trabajo individual, cuando un alumno interactúa consigo mismo para resolver una tarea.

La Función se inspira en las funciones de la demostración matemática en de Villiers (1993, p. 18): verificación, explicación, comunicación, descubrimiento y sistematización. Al igual que este autor, consideramos que la argumentación en el aula de matemáticas no tiene la única función de establecer la plausibilidad de cierta posición respecto de la afirmación de un hecho matemático y que resulta crucial en la construcción y organización del conocimiento, y en la conformación de la disciplina (Cornejo-Morales et al., 2021). Estas funciones se han adaptado para responder a para qué se argumenta: verificar, cuando se establece el valor epistémico de una afirmación dentro de un sistema compartido de conocimientos, como se ha indicado; explicar, cuando se busca dar a comprender las nociones y relaciones matemáticas que están detrás de una afirmación a partir de ideas conocidas; comunicar, cuando se dan a conocer ideas (razonamientos, procedimientos, estrategias, técnicas o definiciones) sobre un tema matemático para dialogar; descubrir, cuando se llegan a establecer nuevas relaciones o comprensiones a nivel local (asociado a la tarea que se está resolviendo); y sistematizar, cuando se llegan a establecer nuevas relaciones o comprensiones a nivel global (cuando la idea se generaliza y aplica a otros casos posibles). En nuestro estudio, a pesar de que no es una función de la demostración en de Villiers (1993), se toma una sexta función de la argumentación: refutar. Esta función se ha conceptualizado, inicialmente, como una acción de carácter complejo que se relaciona con discutir, negociar y contrastar ideas matemáticas o bien rechazar las razones dadas por otro.

El Carácter responde a cómo argumentan los alumnos. Se basa en las ideas de Krummheuer (2013, p. 252), quien identifica dos tipos de argumentación en los primeros años: diagramática y narrativa. La argumentación diagramática usa recursos materiales o pictóricos que 'materializan' los elementos que se ponen en juego en la conversación, mientras que la argumentación narrativa requiere de una narración, a través de la que se establece una 
secuencia entre afirmaciones, donde unas funcionan como causas de otras (Cornejo-Morales y Goizueta, 2019; Krummheuer, 2013).

Las Conexiones interdisciplinares responden a sobre qué se argumenta y a relaciones entre las disciplinas sobre las que se habla. En nuestro caso es una combinación entre ciencias y matemáticas en una actividad STEM. Describimos estos conocimientos en términos de estándares de contenidos o de técnicas y procedimientos de los alumnos para argumentar.

\section{Método}

Se ha llevado a cabo una investigación cualitativa etnográfica (McMillan y Schumacher, 2005) para describir la argumentación de los alumnos en una actividad STEM en una clase de 20 alumnos de 3. ${ }^{\circ}$ de Educación Infantil (5-6 años) del colegio público de Sigüeiro, en Oroso (A Coruña). Todos los alumnos, excepto uno, han asistido al mismo centro y con la misma maestra desde primer curso. Son alumnos que, en general, tienen un dominio adecuado de los conocimientos del nivel acerca de habilidades como la lectura y la escritura.

\subsection{Descripción de la actividad STEM}

La actividad para el aula está vinculada con el entorno e integra conocimientos de matemáticas y ciencias en el marco de la educación STEM (por las siglas en inglés, Science, Technology, Engineering, Mathematics), difundido a través del Informe Rocard (Rocard et al., 2007). Esta actividad se focaliza en la magnitud de la temperatura, a raíz del temporal de frío que afectó a Galicia en noviembre de 2019. Los periódicos y telediarios se invadieron de noticias sobre el frío, nevadas y disminuciones de temperatura y, debido al interés de los alumnos por el tema, en clase surge la pregunta ¿cuánto frío hace? Ante la falta inicial de respuestas, se introduce el uso del termómetro. Se llevan a cabo sesiones en seis días donde se trabajan aspectos ligados a la temperatura: conocimientos previos acerca de esta magnitud; relación con los números para expresar la medida de la temperatura; comportamiento de la temperatura del agua (fría, caliente, templada); dibujo de un termómetro con la serie numérica que gradúa la temperatura y la correlación entre orden de los números de menor a mayor y gradación de frío a caliente, estableciendo una conexión entre "números pequeños/frío" y "números grandes/calientes", pasando por "templado" (Figura 3).

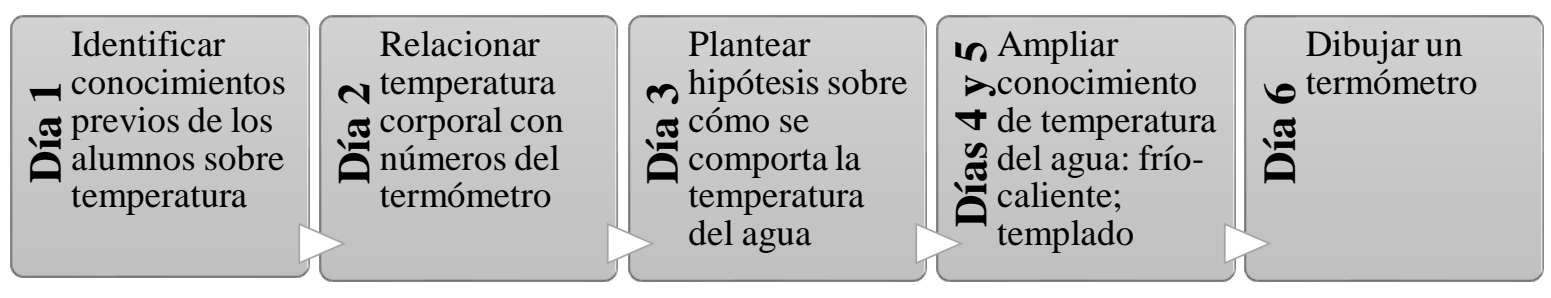

Figura 3. Secuencia de tareas de la actividad STEM

Para el desarrollo de las sesiones se usan diversas estrategias según la sesión: organización en gran grupo o en equipos de trabajo de 4-5, diálogo entre iguales y con la maestra, etc.; junto con diversos recursos y materiales según la sesión: vasos, agua, termómetros, lápiz y papel para anotar, mural y rotuladores para dibujar el termómetro, etc.

La actividad pretende promover la alfabetización STEM de los alumnos proporcionando herramientas que permitan identificar y aplicar, progresivamente, tanto conocimientos clave 
como formas de hacer, pensar, hablar y sentir de la ciencia y de la matemática, de maneras más o menos integradas. Se pretende ayudar a comprender, decidir y/o actuar ante problemas complejos y a construir soluciones creativas e innovadoras, aprovechando las sinergias personales y las tecnologías disponibles, y de forma crítica, reflexiva y con valores (Couso, 2017). Aquí, la argumentación adquiere especial protagonismo.

\subsection{Modelo de análisis de la argumentación: la Situación Argumentativa en Conexión interdisciplinar}

Las seis sesiones de la actividad (Figura 3) se registran en video y transcriben. Tras cada sesión, los autores del artículo analizan el vídeo correspondiente para identificar episodios de interés. En cada episodio se identifica su inicio y final, que se delimitan cuando comienza y finaliza una tarea, cuando se cambia de tema o actividad de la clase y cuando los alumnos trabajan de forma individual. Cada episodio se codifica con $\mathrm{S}$ (n. ${ }^{\circ}$ de sesión); E (n. ${ }^{\circ}$ de episodio de la sesión). Por ejemplo, el código del quinto episodio de la primera sesión es S01E05. Luego, se reconstruye el argumento del episodio, identificando dos elementos centrales: la posición de los alumnos en relación con una afirmación dada y las razones que dan cuenta de la posición. Finalmente, se identifican y describen los elementos de la SAC presentes.

Para resguardar la coherencia en nuestro análisis, se decide identificar solo los episodios de interés en gran grupo o en asamblea, a fin de comparar momentos con características similares de interacción. Para ejemplificar el análisis a partir de la SAC (Figura 2) se ha seleccionado S01E01 (Tabla 1). La maestra plantea la pregunta ¿qué temperatura tenéis? para identificar conocimientos de los alumnos sobre el concepto de temperatura. El episodio termina cuando, en gran grupo, se formula otra pregunta donde se centra el foco.

\section{Tabla 1. Episodio de interés SO1E01}

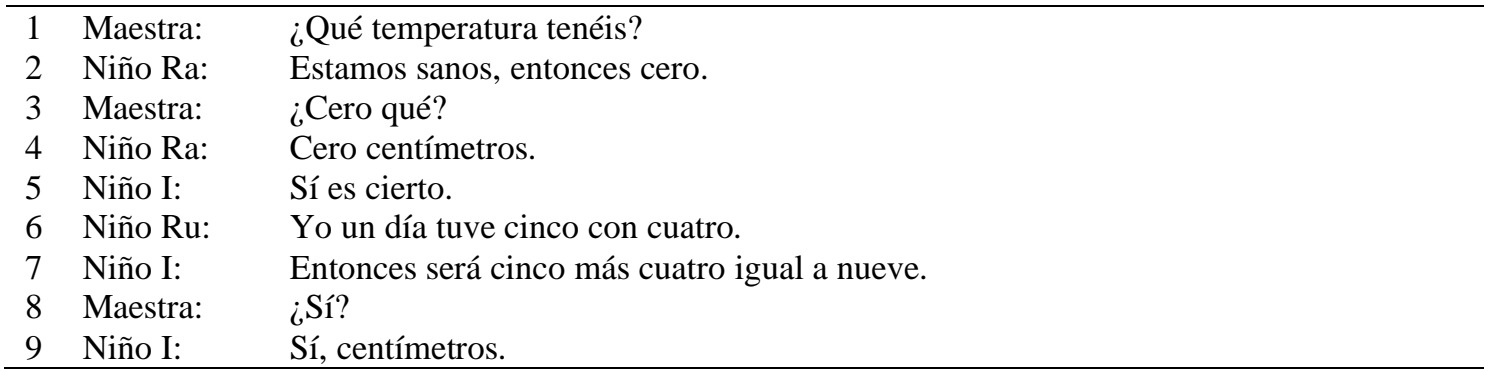

Primero se identifica el argumento del episodio mostrando posición y razones. El Niño Ra formula el Argumento de esta SAC sin ser solicitado por la maestra, aunque motivado por su pregunta. En su Argumento [2], de manera concatenada, el alumno establece una relación directa entre estar sano y tener temperatura. Indica cuánta temperatura (número) se tendría: estar sano implica no tener temperatura o tener cero centímetros (grados) (Figura 4).

El elemento Interacción es de Gran Grupo, guiado por la maestra a través de preguntas orientadas a promover la argumentación de los alumnos y el planteamiento de hipótesis alrededor de la pregunta central e inicial del episodio. 


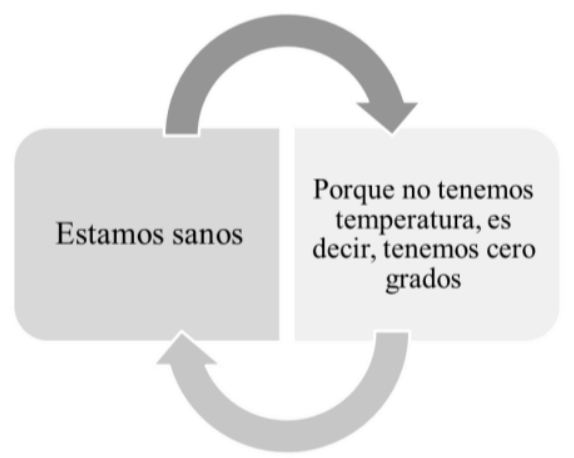

Figura 4. Reconstrucción Argumento central S01E01

La Función que predomina en la argumentación es Explicar. Niño Ra parece utilizar sus ideas sobre el cero y la ausencia de cantidad en relación con la temperatura para justificar su posición respecto de su estado de salud y producir las razones asociadas. El alumno establece relaciones entre sus ideas sobre el cero y la ausencia de temperatura (como signo de salud) para intentar explicar y dar respuesta a la pregunta de la maestra.

El Carácter de la argumentación es Narrativo. El alumno que formula el argumento no hace uso de ningún material concreto para producirlo. Su argumento, breve, establece una relación clara y secuenciada entre la temperatura de una persona, su estado de salud y el número que representaría aquel estado.

Las Conexiones Interdisciplinares tienen que ver con la falta de fiebre como signo de salud y el número que permitiría identificarla. Las ideas matemáticas previas sobre el cero permiten que el alumno formule un argumento sobre la temperatura y el estado de salud.

A partir del análisis de cada elemento, la Figura 5 corresponde a la SAC del episodio seleccionado para mayor detalle en este artículo.

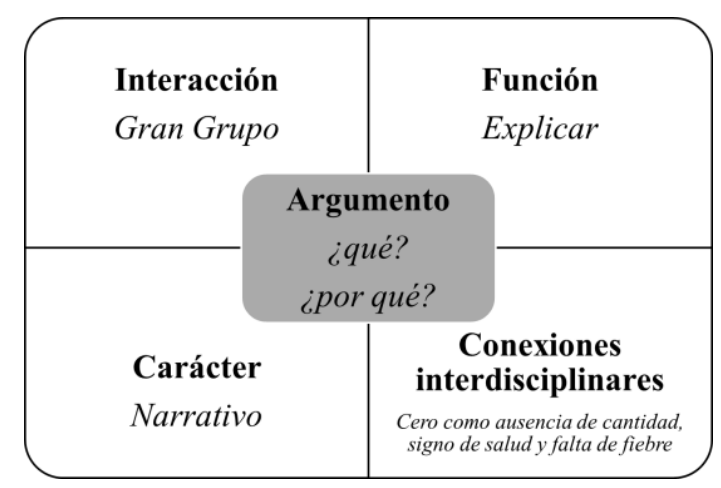

Figura 5. Situación Argumentativa en Conexión interdisciplinaria para S01E01

\section{Identificación de los episodios de interés}

Para determinar los episodios de interés se identifica su argumento a partir de la posición adoptada por los alumnos y las razones que muestran esta posición. Se presentan los nueve episodios de interés identificados, salvo el de la primera sesión (S01E01), descrito en la sección anterior para ejemplificar el análisis a partir de la SAC. En esta primera sesión se realiza 
una primera lluvia de ideas para hacer emerger conocimientos de los alumnos sobre la temperatura. En este primer momento, los alumnos vinculan "tener temperatura", es decir, tener un número en el termómetro, con estar enfermo; y "no tener temperatura", es decir, tener un 0 en el termómetro, con estar bien de salud. Se observa que todavía no conocen la unidad de medida de la temperatura (grado) y utilizan otras magnitudes como la longitud.

Al final de la primera sesión, la maestra propone que se midan la temperatura corporal en casa para que así utilicen el termómetro. El segundo episodio de interés (S02E01) se desarrolla el segundo día, a partir de la medición y registro de la temperatura corporal de cada alumno en casa. Con estos datos, la maestra retoma en asamblea la pregunta del día anterior (“¿qué temperatura tenéis?”). Los alumnos cambian sus ideas, estableciendo una nueva relación: "Cero" no indica estar sano, sino que implicaría estar muerto (Tabla 2).

Tabla 2. Episodio de interés SO2E01

\begin{tabular}{lll}
\hline 1 & Maestra: & Ayer quedamos en que estabais sanos y teníais cero centímetros. \\
2 & Niño Ra: & ¡No!, si tenemos cero estaríamos muertos. \\
3 & Maestra: & ¿Medisteis la temperatura en casa? \\
4 & Niños/as: & ¡Sí! \\
\hline
\end{tabular}

En S02E02 (Tabla 3), aparecen nuevos números: algunos alumnos son capaces de leer números de dos cifras (e. g. "treinta y seis y algo"), mientras que otros los referencian cifra a cifra (e. g. "un tres y un siete"). Aunque siguen sin conocer la unidad de medida de la temperatura, dejan de nombrarla con unidades de otras magnitudes.

\section{Tabla 3. Episodio de interés S02E02}

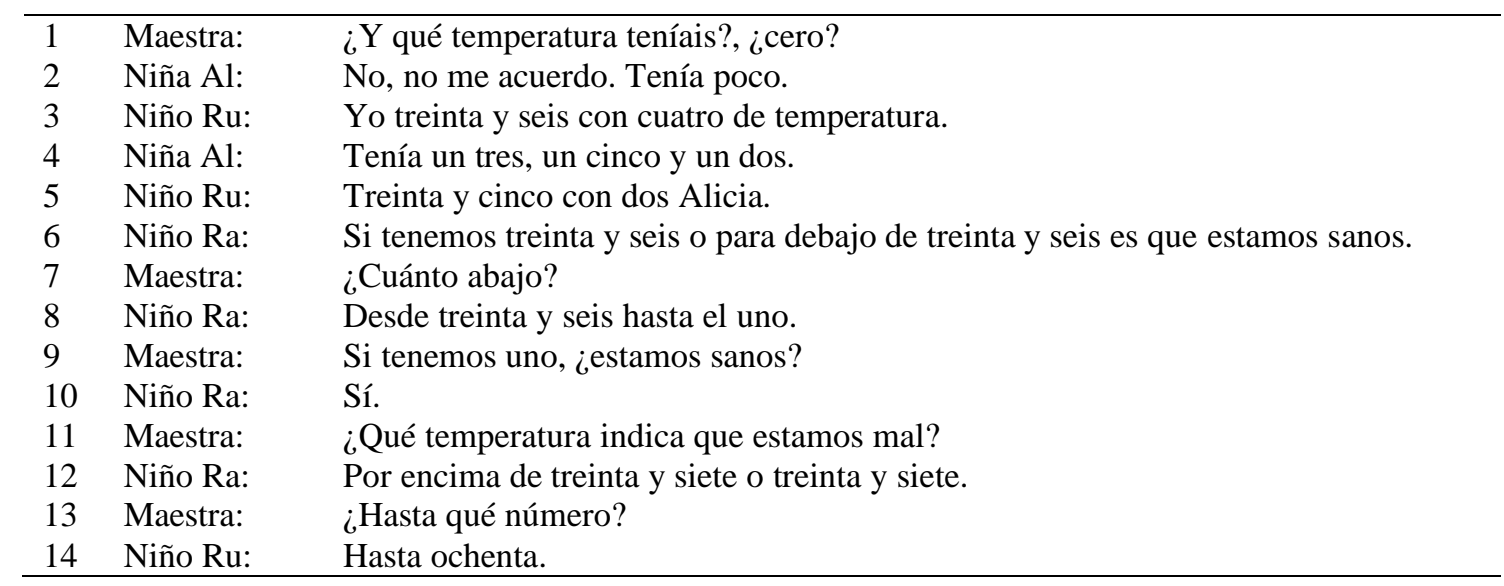

La maestra plantea preguntas para que surjan ideas para representar un termómetro. En este sentido, S02E03 (Tabla 4) se inicia con: “¿alguna vez tuvisteis ochenta de temperatura?”.

El tercer día se plantea una tarea a partir de la temperatura del agua. Dicha tarea promueve el planteamiento de hipótesis sobre qué ocurre, con el paso del tiempo, a la temperatura del agua que hay en dos vasos: uno con agua caliente del microondas $\left(80^{\circ} \mathrm{C}\right)$ y otro con agua fría de la nevera $\left(0^{\circ} \mathrm{C}\right)$, como se aprecia en S03E01 (Tabla 5). 
Tabla 4. Episodio de interés SO2E03

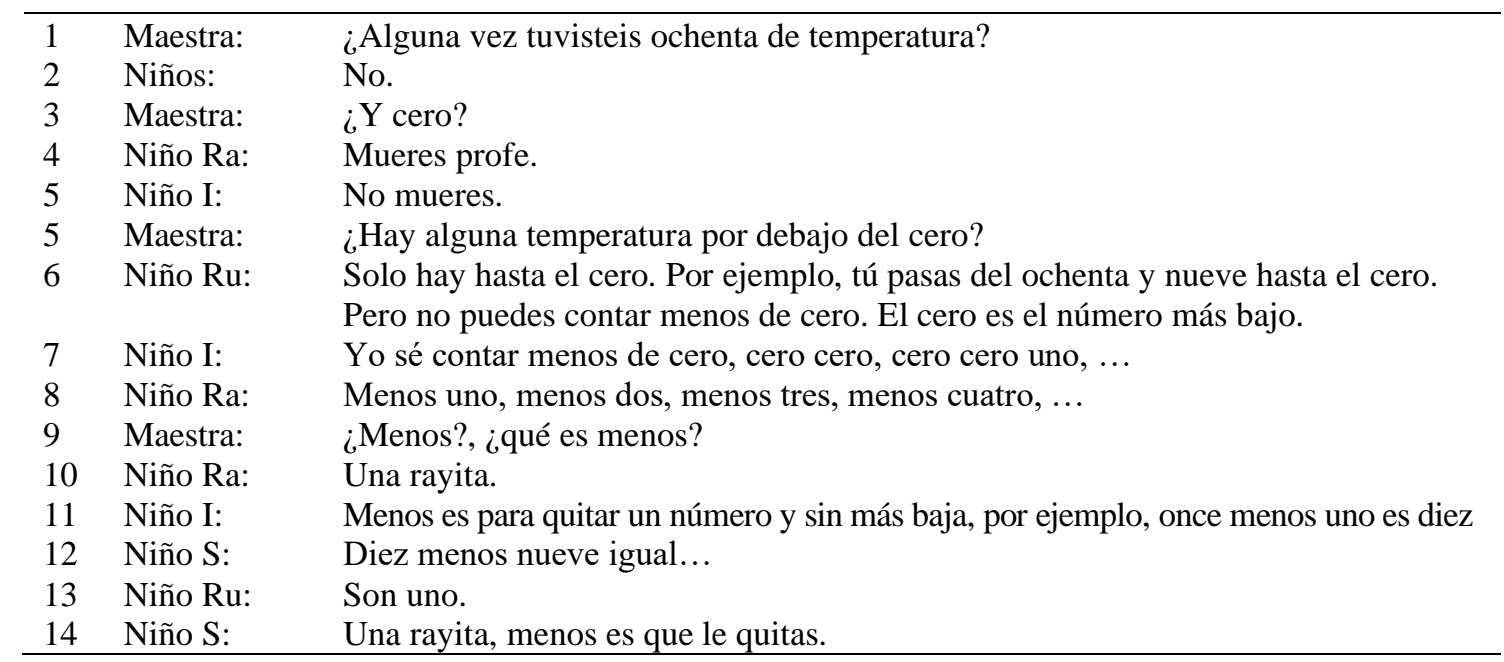

Tabla 5. Episodio de interés SO3E01

\begin{tabular}{lll}
\hline 1 & Niño Ru: & Que el caliente enfría y el frío calienta. \\
2 & Niño I: & Yo creo que el caliente enfría y el frío pasa a estar templado. \\
3 & Niño A: & El vaso frío pasa a caliente y el caliente a menos caliente. \\
4 & Niña Ar: & El frío se calienta y el caliente se enfría. \\
\hline
\end{tabular}

Durante una hora y media, los alumnos toman ocho veces la temperatura del agua de los dos vasos y registran los datos en una tabla de doble entrada (Figura 6).

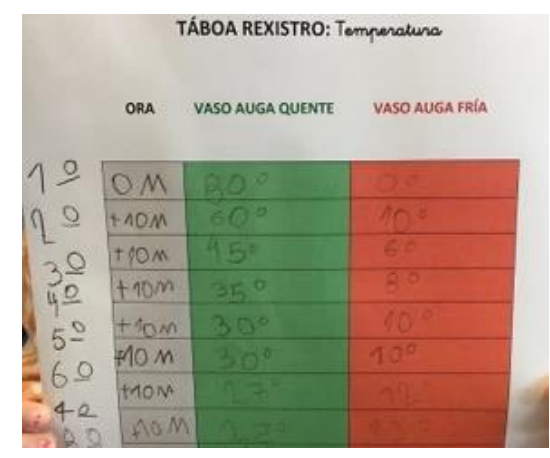

Figura 6. Registro de la temperatura

Las primeras tomas se hacen en pareja para apoyarse, por si no se domina el manejo del instrumento o la identificación de dígitos, pero el registro es individual (Figura 7).

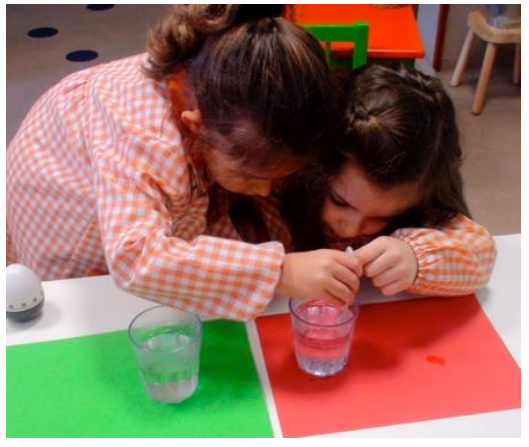

Figura 7. Toma de temperatura del agua 
Una vez realizados los ocho registros se hace una puesta en común para hablar de los resultados, fundamentarlos y establecer relaciones. Los alumnos se dan cuenta de que el agua caliente pasa de un estado inicial de $80{ }^{\circ} \mathrm{C}$ en la primera toma a $25^{\circ} \mathrm{C}$ en la octava, mientras que el agua fría pasa de $0{ }^{\circ} \mathrm{C}$ a $13{ }^{\circ} \mathrm{C}$.

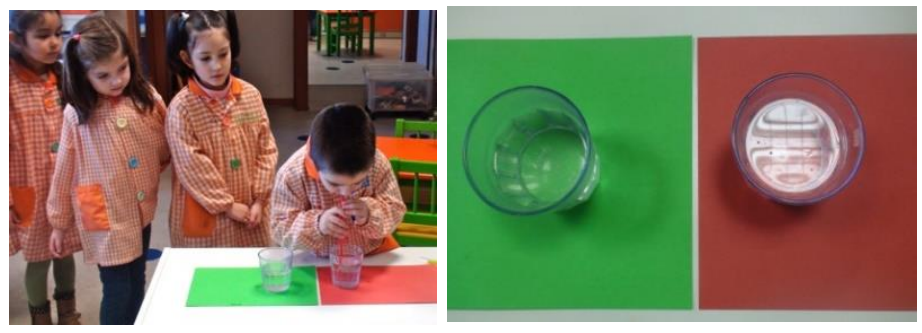

Figura 8. Comprobando la sensación de $14^{\circ}$

A primera hora del cuarto día los alumnos realizan otro registro, a las 20 horas del primero, y observan que el agua de los dos vasos ha alcanzado la misma temperatura $\left(14^{\circ} \mathrm{C}\right)$. La maestra interviene para cuestionar si $14^{\circ} \mathrm{C}$ es frío o caliente y los alumnos, tras varias suposiciones, proponen tocar y probar el agua de los dos vasos para comprobar la sensación (Figura 8). Argumentan que "es frío" usando el lenguaje verbal, en ningún caso el numérico.

Cuatro horas más tarde los alumnos realizan otra medición de la temperatura del agua de los vasos, que no registran en la tabla, y observan que ha aumentado un grado. Atribuyen el aumento a la temperatura ambiental y al efecto de la calefacción. Empiezan a hacer las primeras generalizaciones: relacionan la disminución de números con "enfriar” y el aumento con "calentar". Para comprobar estas ideas, los alumnos sugieren poner los dos vasos al lado de un radiador durante una hora y realizar otra medición. De nuevo, tocan y prueban el agua $\mathrm{y}$, al comprobar que no hay variaciones, tampoco registran el dato y proponen poner los dos vasos encima del radiador durante dos horas. Todos experimentan la sensación y una pareja hace la medición, que es de $16^{\circ} \mathrm{C}$. En gran grupo analizan los diez registros y concluyen que, cuanto más tiempo encima del radiador, más caliente queda el agua.

El quinto día se lleva a cabo una asamblea o puesta en común para hacer emerger ideas sobre la temperatura del agua de los vasos que se colocaron encima del radiador. La maestra retoma lo ocurrido y plantea: “con el paso del tiempo, ¿qué ocurre?” En sus respuestas, los alumnos usan palabras e incorporan números y la unidad de medida de la temperatura. Luego, como se observa en S06E01 (Tabla 6), uno de los alumnos toma la temperatura del agua e indica que no alcanza los $30^{\circ} \mathrm{C}$, que está por debajo, y surge la duda de si es fría o caliente.

\section{Tabla 6. Episodio de interés S06E01}

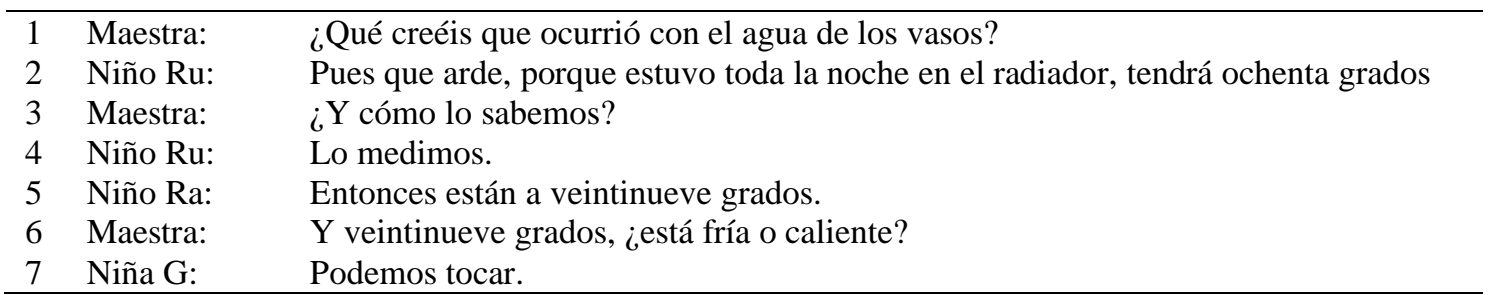


Tabla 7. Episodio de interés SO7E01

\begin{tabular}{lll}
\hline 1 & Maestra: & $\begin{array}{l}\text { Pues ahora en el papel, ¿qué números ponemos?, ¿cuál será el más grande?, } \\
\text { ¿cuál será el más pequeño? }\end{array}$ \\
2 & Niño Ru: & El cero abajo y el cien arriba, igual doscientos, no cien arriba. \\
3 & Maestra: & El cien arriba, y cien grados ¿cómo está? \\
4 & Niño I: & Caliente. \\
5 & Niño Ra: & Y cero abajo frío. \\
6 & Maestra: & Genial, ¿lo registráis? \\
7 & Maestra: & ¿Alguna otra temperatura? \\
8 & Niño Ru: & Veintisiete es templado. \\
9 & Maestra: & ¿Lo registras? \\
10 & Niño X: & Y catorce grados es fría. \\
\hline
\end{tabular}

Entre todos van complementando datos y usan números para argumentar ideas. Tras la experimentación, las opiniones siempre se declinan por templada/fría. Al cabo de una hora más y una nueva toma, observan que la temperatura del agua se incrementa un grado, pero que todavía la sienten templada, concluyendo que entre $29^{\circ} \mathrm{C}$ y $30^{\circ} \mathrm{C}$ el agua está templada.

El sexto y último día se propone representar, entre todos, un termómetro en un mural registrando sus números con colores: frío (rojo), templado (azul) y caliente (verde). Se dibuja una línea vertical; a través del diálogo, se va cubriendo con los números que van indicando los niños y niñas, tal como muestran S07E01 (Tabla 7) y la Figura 9.

A partir de la mediación de las preguntas de la maestra, se establece el intervalo del termómetro, acotando como la temperatura más baja $0{ }^{\circ} \mathrm{C}$ y la más alta $100{ }^{\circ} \mathrm{C}$. Seguidamente, todos participan aportando sus números $\mathrm{y}$, teniendo en cuenta la comprensión de la serie numérica, se observa cómo algún alumno aporta algún otro dato que rompe con la serie o continúa en la línea del dato anterior registrado, como muestra S07E02 (Tabla 8).

Tabla 8. Episodio de interés S07E02

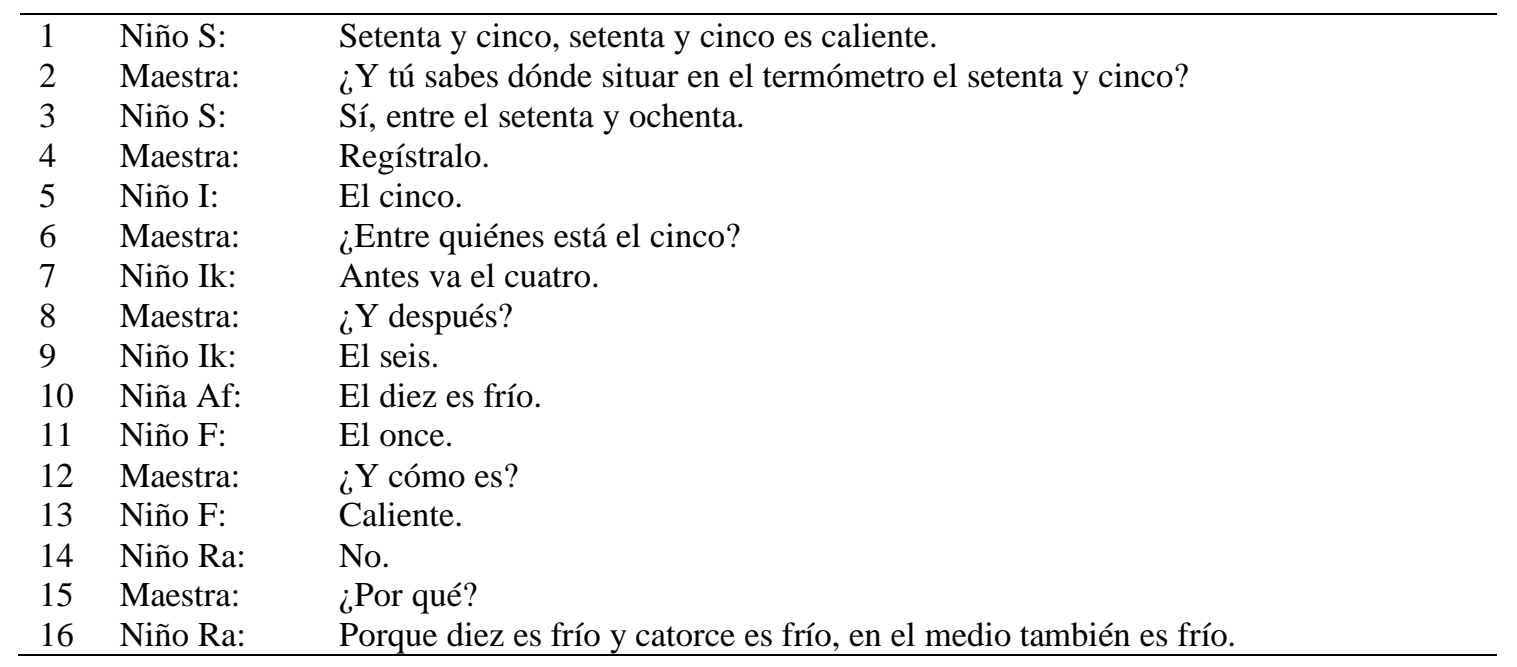

Cuando se pregunta a los alumnos por más números, aparece el 80 que sitúan como caliente, y el 1 que determinan frío. Aparecen después los números 2, 3, 4 y 6, que no dudan que son fríos. Los extremos del intervalo quedan bien definidos por números $\mathrm{y}$, al preguntar por otros números, asocian el 27 a templado. Entonces, la maestra pide relacionar las ideas de caliente y templado y los rangos de temperatura, como muestra S07E03 (Tabla 9). 
Tabla 9. Episodio de interés S07E03

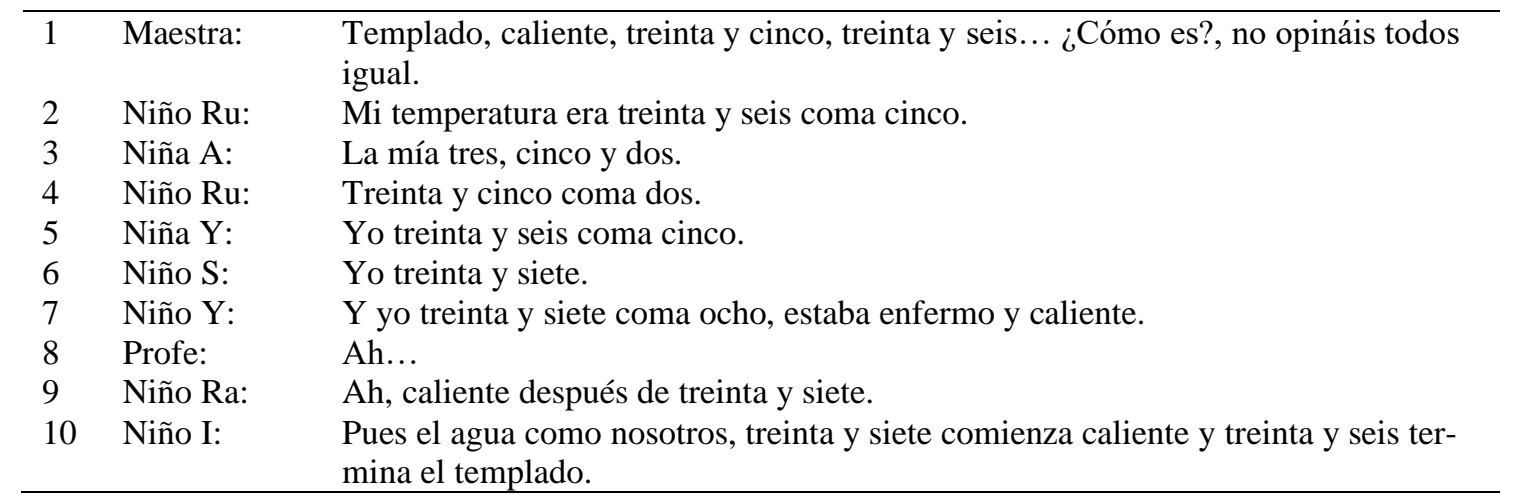

En síntesis, se observa cómo, a través de la experimentación y de números conocidos, los alumnos van estableciendo nuevas relaciones y acotan nuevos datos. Dato a dato, construyen y rellenan el termómetro de datos numéricos (Figura 9).

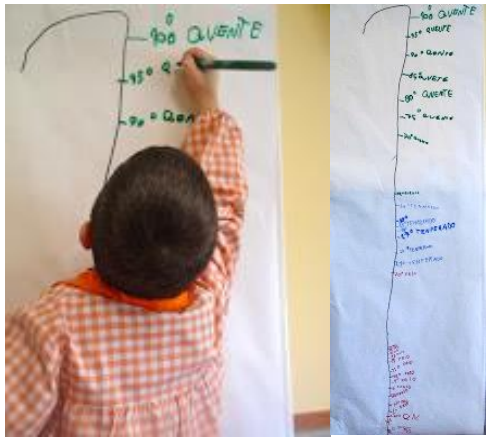

Figura 9. Proceso de representación del termómetro

\section{Análisis de los elementos de la Situación Argumentativa en Conexión Interdisciplinar}

La Tabla 10 muestra la distribución de los elementos de la SAC en la secuencia de tareas analizada. No se presenta la interacción de cada episodio porque todas son de gran grupo.

Tabla 10. Distribución de episodios y principales características

\begin{tabular}{ccc}
\hline Episodio & $\begin{array}{c}\text { Función de la } \\
\text { Argumentación }\end{array}$ & $\begin{array}{c}\text { Carácter de la } \\
\text { Argumentación }\end{array}$ \\
\hline S01E01 & Explicar & Narrativo \\
S02E01 & Sistematizar & Narrativo \\
S02E02 & Explicar & Narrativo \\
S02E03 & Explicar & Narrativo \\
S03E01 & Descubrir & Narrativo \\
S06E01 & Explicar - Verificar & Narrativo \\
S07E01 & Sistematizar & Diagramático \\
S07E02 & Refutar & Narrativo \\
S07E03 & Descubrir & Narrativo \\
\hline
\end{tabular}




\section{Sobre los argumentos}

Los argumentos identificados en esta secuencia de tareas, y posteriormente reconstruidos por los autores, se caracterizan por ser breves, responder a una pregunta de la maestra y usar expresiones clave para argumentar. Los alumnos usan expresiones que muestran la relación entre los elementos constitutivos de un argumento: posición y razones que dan cuenta de esta posición. Por ejemplo, en S01E01, el argumento del alumno es: "Estamos sanos, entonces tenemos cero". En este argumento, el alumno usa la palabra entonces para establecer una relación entre estar sano y la razón que da cuenta de esa afirmación. Para él, estar sano implica no tener temperatura, es decir, cero grados. Esta palabra es clave porque muestra la relación entre no tener temperatura y estar sano, como una implicación o relación directa.

A lo largo de la secuencia, los alumnos también usan ejemplos y contraejemplos para argumentar. En S02E03, la maestra pregunta qué pasaría si tenemos $0{ }^{\circ} \mathrm{C}$ de temperatura y uno de los alumnos indica que, si esto pasara, moriríamos. Cuando la maestra pregunta sobre temperaturas "menos que cero", el mismo alumno replica que no hay, pues "solo hay hasta el cero. Por ejemplo, tú pasas del ochenta y nueve hasta el cero. Pero no puedes contar menos de cero. El cero es el número más bajo". En su argumento, el alumno establece dos relaciones. La primera se observa cuando dice por ejemplo y enfatiza los intervalos numéricos entre los que fluctúa la temperatura. En este caso, el alumno produce un contraejemplo para argumentar, basado en su experiencia y con los números. Aún no concibe la idea de que algo pueda ser menor que cero. Luego, usa la palabra pero para conectar estas dos ideas (se puede tener temperatura entre $0^{\circ}$ y $89^{\circ}$ pero no menor que $0^{\circ}$ ) ya enfatizar lo que está diciendo.

A través de la lengua, los alumnos formulan hipótesis basadas en su experiencia y en las relaciones que establecen entre la experiencia de aprendizaje y sus ideas. En S02E02, un alumno hipotetiza sobre los intervalos de temperatura y su relación entre estar sanos o enfermos, y enfatiza una nueva relación: "si tenemos treinta y seis grados o por debajo de treinta y seis grados es que estamos sanos". En este argumento, el alumno usa las expresiones $s i$ tenemos y por debajo de treinta y seis grados para destacar que hay criterios al establecer el estado de salud de una persona. En S06E01, el alumno formula una hipótesis sobre qué pasaría con la temperatura del agua que estuvo toda la noche en el radiador diciendo "pues que arde, porque estuvo toda la noche en el radiador, tendrá ochenta grados”. En este argumento porque es conjunción causal que introducen razones sobre esta afirmación.

En S07E03, el alumno establece relaciones entre experiencias trabajadas en la secuencia, indicando que con la temperatura del agua pasa lo mismo que con la temperatura corporal, como nosotros. El alumno enfatiza que "pues el agua como nosotros, treinta y siete comienza caliente y treinta y seis termina el templado". A medida que avanza la actividad, se sofistican los argumentos debido al uso de la lengua y al establecimiento de conjeturas basadas en la experiencia y en la introducción de aprendizajes desarrollados. Además, la extensión de los argumentos aumenta al avanzar en la secuencia y en la comprensión de los temas abordados.

\section{Sobre la función de la argumentación}

En esta secuencia de tareas, predomina la función Explicar (S01E01, S02E02, S02E03, S06E01), donde los alumnos principalmente describen nociones y relaciones que están detrás de una idea sobre la temperatura, a partir de la experiencia, propiedades y estrategias diversas. 
Solo en S06E01 (Tabla 6) los alumnos argumentan para verificar sus procedimientos, aunque la función Verificar no es predominante como finalidad de la argumentación, sino que actúa subordinada a Explicar. En este episodio, los alumnos verifican [4] usando instrumentos de medición de la temperatura para comprobar si su explicación sobre la temperatura del agua es correcta. La tarea promueve el uso de instrumentos y la experiencia desarrollada incita a medir y verificar la temperatura del agua.

Al avanzar en la secuencia, se evidencian funciones más sofisticadas (Descubrir Sistematizar y Refutar). En S07E02, se observa la función Refutar pues se identifica más de una posición cuando la maestra solicita establecer relaciones entre los números conocidos y experimentados y otros números cercanos a los registrados. Hay alumnos que no están de acuerdo en que $11^{\circ} \mathrm{C}$ es caliente, exponen posiciones al respecto y logran acordar que $11^{\circ} \mathrm{C}$ es frío "porque si diez es frío y catorce es frío, en el medio también es frío". Observamos Descubrir y Sistematizar cuando los alumnos establecen relaciones y comprensiones, locales y globales, usando técnicas, procedimientos o estrategias al responder a una tarea.

\section{Sobre el carácter de la argumentación}

La argumentación en esta secuencia se caracteriza por ser narrativa. Por un lado, esto implica que los alumnos hacen uso de la oralidad para argumentar y no recurren a otros recursos disponibles (termómetros, dibujos, secuencias numéricas). Se observan, por tanto, un "abandono" del recurso concreto y la capacidad de decir con palabras aquello que están pensando o viendo en la experiencia de aprendizaje. Por otro lado, esta secuencia fue pensada para promover el tránsito desde lo diagramático a lo narrativo y desarrollar la narratividad de los alumnos a través de buenas preguntas y actividades de aprendizaje desafiantes, donde el recurso no sea suficiente para formular un argumento. En esta secuencia se tensionan las ideas de los alumnos a través de las tareas y a través de la promoción de espacios de diálogo.

\section{Sobre las conexiones interdisciplinares}

Esta secuencia de tareas responde a las características del aprendizaje en los primeros años y promueve el desarrollo de conexiones entre disciplinas. Para lograrlo, se proponen tareas que abordan contenidos matemáticos y científicos a través de la experimentación, sin identificar con precisión diferencias entre la naturaleza de conocimientos involucrados. El conocimiento científico abordado es la temperatura corporal y la temperatura del agua en estado líquido. Las competencias científicas guardan relación con la formulación de hipótesis y conjeturas, con la experimentación y con el uso de los sentidos e instrumentos de medición. Las ideas matemáticas involucradas son el cero como ausencia de cantidad, la grafía de los números, la ampliación del ámbito numérico, el establecimiento de intervalos (de temperatura), el uso de cuantificadores (mayor, menor, igual), el establecimiento de relaciones de comparación entre los números involucrados y la representación de los números en la recta numérica, entre otros. La argumentación tanto científica como matemática son elementos centrales de esta actividad, donde no interesa si están hablando de ciencias o de matemáticas, sino las relaciones que se establecen entre ambas disciplinas para responder a la problemática propuesta. No interesa que una disciplina predomine sobre la otra o sea más importante; a veces se habla más sobre ciencias al experimentar y en otras ocasiones, se habla más sobre los números y sus relaciones. En esta actividad, pues, las disciplinas se fusionan. 


\section{Consideraciones finales}

En este estudio se ha descrito una actividad STEM en Educación Infantil, de conexiones entre ciencias y matemáticas, y se han analizado las actuaciones de los alumnos en términos funcionales y contextuales de la argumentación en matemáticas, a partir de la Situación Argumentativa en Conexión interdisciplinar (SAC), lo que constituye la principal novedad respecto a Cornejo-Morales et al. (2021), en el que se utilizó como modelo de análisis la SA.

Se ha llevado a cabo una secuencia de tareas para que los alumnos pudieran relacionar la temperatura corporal con los números, a través de la argumentación. Para avanzar en este sentido, la gestión de la maestra ha consistido principalmente en ir planteando preguntas a los alumnos. A raíz de esta gestión, los alumnos han comunicado conocimientos, experiencias y creencias en un marco de interacción en gran grupo y diálogo.

El análisis de la argumentación a partir de la SAC, más allá de centrarse en su estructura y en los tipos de argumento, ha permitido identificar qué se argumenta y por qué, quiénes argumentan, para qué se argumenta, cómo y sobre qué. Se ha evidenciado que los argumentos de los niños se caracterizan por ser breves, responder a una pregunta de la maestra y usar expresiones clave para mostrar la relación entre los elementos constitutivos de un argumento (posición y razones), ya sea a través de ejemplos y contraejemplos o bien del planteamiento de hipótesis. Otro resultado es que los argumentos se van sofisticando progresivamente, en relación con el uso de la lengua, con el establecimiento de conjeturas basadas en la experiencia y la comprensión de los temas abordados.

Respecto a la función de la argumentación, en la actividad STEM analizada predomina Explicar, a modo de descripción de nociones y relaciones que están detrás de una idea sobre la temperatura, a partir de la experiencia, propiedades y estrategias diversas. Se observan también otras funciones más sofisticadas como Descubrir, Sistematizar y Refutar con menor presencia. Interpretando a de Villiers (1993), consideramos que el predominio de Explicar se debe a las características intrínsecas de la secuencia de tareas, en la que se promueven explicaciones sobre un fenómeno y relaciones entre números y temperatura.

Siguiendo con el resto de los elementos de la SAC, el carácter predominante de la argumentación es narrativa (Krummheuer, 2013), lo que evidencia que los alumnos de los últimos años de Educación Infantil van dejando atrás los recursos concretos, es decir, lo diagramático, para dar entrada a la oralidad como principal herramienta para argumentar.

La actividad responde a un planteamiento interdisciplinar que integra conocimientos científicos y matemáticos a través de la experimentación, sin identificar diferencias entre los ámbitos de conocimiento. La argumentación tanto científica como matemática son elementos centrales de la actividad STEM donde interesan las relaciones que se establecen entre disciplinas para encontrar una solución al problema planteado. En el futuro, va a ser necesario diseñar nuevos estudios que, en el marco de las conexiones interdisciplinares, utilicen este modelo de análisis para consolidar los resultados iniciales, que han permitido constatar la presencia de la argumentación en las matemáticas escolares de las primeras edades.

\section{Agradecimientos}

Agencia Nacional de Investigación y Desarrollo de Chile (ANID), a través de Beca de Doctorado Nacional convocatoria 2017 folio 21171532. FEDER/Ministerio de Ciencia, Innovación y Universidades -AEI, Proyecto EDU2017-84979-R. 


\section{Referencias}

Alsina, Á., Maurandi, A., Ferre, E. y Coronata, C. (2021). Validating an instrument to evaluate the teaching of mathematics through processes. International Journal of Science and Mathematics Education, 19, 559-577. https://doi.org/10.1007/s10763-020-10064-y

Artigue, M. y Blomhøj, M. (2013). Conceptualizing inquiry-based education in mathematics. ZDM Mathematics Education, 45, 797-810. https://doi.org/10.1007/s11858-013-0506-6

Balacheff, N. (1999). Is argumentation an obstacle? Invitation to a debate. International Newsletter on the Teaching and Learning of Mathematical Proof, Mai/Juin. http://www.lettredelapreuve.org/OldPreuve/Newsletter/990506Theme/990506ThemeUK.html

Boero, P. (2011). Argumentation and proof: Discussing a "successful" classroom discussion. En M. Pytlak, T. Rowland \& E. Swoboda (eds.), Proceedings of the $7^{\text {th }}$ Congress of the European Society for Research in Mathematics Education (pp. 120-130). ERME.

Boero, P., Douek, N., Morselli, F. y Pedemonte, B. (2010). Argumentation and proof: A contribution to theoretical perspectives and their classroom implementation. En M. Fusaro et al. (Eds.), Proceedings of $34^{\text {th }}$ International Conference of the Psychology of Mathematics Education (Vol. 1, pp. 179-205). PME.

Cornejo-Morales, C. y Alsina, Á. (2020). La argumentación en los currículos de Educación Matemática Infantil. Edma 0-6, 9(1), 12-30.

Cornejo-Morales, C. y Goizueta, M. (2019). El tránsito entre argumentos diagramáticos y narrativos en preescolar. Orientaciones y propuestas. UNO, 85, 28-31.

Cornejo-Morales, C., Goizueta, M. y Alsina, Á. (2021). La Situación Argumentativa: un modelo para analizar la argumentación en educación matemática infantil. PNA, 15(3), 159-185.

Couso, D. (2017). Per a què estem a STEM? Un intent de definir l'alfabetització STEM per a tothom i amb valors. Ciències, 34, 22-30. https://doi.org/10.5565/rev/cien$\underline{\text { cies. } 403}$

De Villiers, M. (1993). El papel y la función de la demostración en matemáticas. Épsilon, 26, 15-30.

Douek, N. (2007). Some remarks about argumentation and proof. En P. Boero (Ed.), Theorems in school: From history, epistemology and cognition to classroom practice (pp. 163-181). Sense Publishers. https://doi.org/10.1163/9789087901691_010

Duval, R. (1999). Argumentar, demostrar, explicar: ¿Continuidad o ruptura cognitiva? Grupo Editorial Iberoamérica.

Godino, J. D., Batanero, C., Cañadas, G. R. y Contreras, J. M. (2015). Articulación de la indagación y transmisión de conocimientos en la enseñanza y aprendizaje de las matemáticas. En B. D’Amore y M. I. Fandiño (eds.), Congreso Internacional Didáctica de la Matemática (pp. 249-269). Universidad de la Sabana. 
Goizueta, M. (2019). Epistemic issues in classroom mathematical activity: There is more to students' conversations than meets the teacher's ear. The Journal of Mathematical Behavior, 55, 1-11. https://doi.org/10.1016/j.jmathb.2019.01.007

Goizueta, M. y Solar, H. (2019). Relaciones entre la argumentación en el aula de matemáticas y la mirada profesional del profesor. En R. Olfos, E. Ramos y D. Zakaryan (eds.), Aportes a la práctica docente desde la didáctica de la matemática (pp. 241-280). Graó.

Inglis, M., Mejía-Ramos J. P. y Simpson, A. (2007). Modeling mathematical argumentation: The importance of qualification. Educational Studies in Mathematics, 66, 3-21. https://doi.org/10.1007/s10649-006-9059-8

Knipping, C. (2008). A method for revealing structures of argumentation in classroom proving processes. ZDM Mathematics Education, 40, 427-441. https://doi.org/10.1007/s11858-008-0095-y

Krummheuer, G. (1995). The ethnography of argumentation. En P. Cobb y H. Bauersfeld (eds.), The emergence of mathematical meaning: Interaction in classroom cultures (pp. 229-269). Lawrence Erlbaum.

Krummheuer, G. (2013). The relationship between diagrammatic argumentation and narrative argumentation in the context of the development of mathematical thinking in the early years. Educational Studies in Mathematics, 84, 249-265.

https://doi.org/10.1007/s10649-013-9471-9

Kuhn, D. (1991). The skills of argument. Cambridge University Press. https://doi.org/10.1017/CBO9780511571350

McMillan, J. H. y Schumacher, S. (2005). Investigación educativa. Pearson Addison Wesley.

National Council of Teachers of Mathematics [NCTM] (2003). Principios y estándares para la educación matemática. Thales.

Niss, M. (2002). Mathematical competencies and the learning of mathematics: The Danish Kom Project. Roskilde University Press.

Rocard, M., Csermely, P., Jorde, D., Lenzen, D., Walwerg Henriksson, H. Y. y Hemmo, V. (2007). Science education now: A renewed pedagogy for the future of Europe. Office for Official Publications of the European Communities.

Schwarz, B. B., Neuman, Y., Gil, J. y Ilya, M. (2003). Construction of collective and individual knowledge in argumentative activity. The Journal of the Learning Sciences, 12(2), 219-256. https://doi.org/10.1207/S15327809JLS1202_3

Solar, H. y Deulofeu, J. (2016). Condiciones para promover el desarrollo de la competencia de argumentación en el aula de matemáticas. Bolema, 30(56), 1092-1112. https://doi.org/10.1590/1980-4415v30n56a13

Toulmin, S. (1958). The uses of argument. Cambridge University Press. 


\section{Referencias de los autores}

Ángel Alsina, Facultad de Educación y Psicología, Universidad de Girona (España). angel.alsina@udg.edu

Claudia Cornejo-Morales, Instituto de Matemáticas, Pontificia Universidad Católica de Valparaíso (Chile). claudia.cornejo.morales@gmail.com

María Salgado, Facultad de Ciencias de la Educación, Universidad de Santiago de Compostela (España). maria.salgadosomoza@ hotmail.com 


\title{
Argumentation in early school mathematics: Analysis of a STEM activity using the Argumentative Situation in Interdisciplinary Connection
}

\author{
Ángel Alsina, Universidad de Girona \\ Claudia Cornejo-Morales, Pontificia Universidad Católica de Valparaíso \\ María Salgado, Universidad de Santiago de Compostela
}

This article analyses argumentation in the framework of a STEM activity implemented in an Early Childhood Education classroom where mathematics and science knowledge are integrated. Given the characteristics of the activity, a variant of the Argumentative Situation (SA) is used as analytical model to describe the characteristics of argumentation, namely, the Argumentative Situation in Interdisciplinary Connection (SAC). The SA describes and characterises argumentation in early mathematics classrooms through five structural elements: 1) Argument (what is being argued and why?); 2) Interaction (who is arguing?); 3) Function (what is being argued for?); 4) Character (how is the argument being made?); and 5) Mathematics (what is it argued about?), while the SAC replaces the Mathematics element with Interdisciplinary Connections, which responds to what is being argued about as well as to the relationships between the disciplines under discussion. Based on qualitative ethnographic research, we analyse the argumentation of 20 children in the 3rd year of Early Childhood Education (5-6 years old) at the Siguieiro public school in Oroso (A Coruña, Spain). The data analysis shows that in the sequence of tasks, the main function of the argumentation is to explain and the character is narrative: the arguments are short and concise, they answer a specific question and use key expressions to show the relationship between its constituent elements. In addition, the activity responds to an interdisciplinary approach that integrates scientific and mathematical knowledge through experimentation, without identifying differences between the fields of knowledge. Both scientific and mathematical argumentation are central elements of the STEM activity, in which the relationships between disciplines are of interest in order to find a solution to the problem given. In the future, it will be necessary to design new studies that, within the framework of interdisciplinary connections, use this analytical model to consolidate our initial results. We have importantly confirmed the presence of argumentation in early school mathematics. 